\title{
Using Orthogonal Combined Signals in Broadband ADCP for Improving Velocity Measurement
}

\author{
Yujing Lin, Fei Yuan* * and En Cheng \\ Key Laboratory of Underwater Acoustic Communication and Marine Information Technology, \\ Ministry of Education, Xiamen Universit, Xiamen 361005, China; linyujing@stu.xmu.edu.cn (Y.L.); \\ chengen@xmu.edu.cn (E.C.) \\ * Correspondence: yuanfei@xmu.edu.cn; Tel.: +86-1330-602-9530
}

Received: 25 April 2020; Accepted: 1 June 2020; Published: 19 June 2020

\begin{abstract}
Broadband Acoustic Doppler Current Profiler (BBADCP) is a widely used technology in velocity measurements. To adapt to the varied water environment and different measurement requirements, flexible tuning of transmitted signal parameters will improve the feasibility and accuracy of velocity measurement. Compared with the conventional signal, the orthogonal combined signal designed in this paper can generate a wealth of signal combination examples and improve the accuracy of the velocity measurement under the same conditions. The proposed orthogonal combined signal consists of two orthogonal sub-signals with a symmetrical spectrum. Each is designed based on time delay to eliminate or weaken the current velocity ambiguity. Then, the processing method of the received signal when the pulse signals are the same or different coded signal is discussed. The numerical simulation results show that, when using the proposed method, the standard deviation of the estimated current velocity has different degrees of reduction at different current velocities. Our simulation also shows that, compared to the convention method, the proposed method can improve the SNR by $10 \mathrm{~dB}$. This can help significantly increase the scope of the configuration.
\end{abstract}

Keywords: ADCP; current velocity; transmitted signal; orthogonal combined signal

\section{Introduction}

Acoustic Doppler Current Profilers (ADCPs) are widely used in the current velocity measurement by using Doppler principle, which has the characteristics of the undisturbed flow field and no mechanical inertia. Doppler measurement techniques can be divided into three types: pulse-incoherent Doppler, pulse-coherent Doppler, and coded-pulse broadband acoustic Doppler [1].

Pulse-incoherent Doppler current measurement technique is also called narrow-band current measurement technique [2]; the sensor transmits a fixed single frequency sine wave signal. Only when the signal-noise ratio (SNR) is higher than a certain threshold can it show better flow measurement performance. Pulse-coherent Doppler technique is to measure the current velocity by sending two single frequency sine wave signals successively. Compared with pulse-incoherent Doppler, it can obtain a smaller depth unit and a higher layer thickness resolution due to the shorter pulse width [3]. Coded-pulse broadband acoustic Doppler technology, also referred to as Broadband ADCPs (BBADCPs), effectively combines the advantages of the above two techniques [4]. The transmitted pulse signal is modulated by Pseudo-Noise (PN) code, which can improve the measurement accuracy of current velocity and low range resolution [5-7]. Thus, BBADCPs are commonly used in current measurement.

Generally, the Doppler shift is estimated by extracting the phase of the autocorrelation function of the received complex baseband signal [8]. The pulse-pair method, also known as the covariance method, was first proposed by Miller in 1972, and has become a popular frequency estimation algorithm 
used in BBADCP, due to its less computation and higher accuracy [8-10]. S. S. Abeysekera [11] compared the pulse-pair method with Fast Fourier Transformation (FFT) and concluded that the operation of the pulse-pair method is less than that of FFT. In 2001, the frequency estimation method based on the Discrete Fourier Transform (DFT) was proved to perform better than the pulse-pair method in low SNR [12]. However, realized on hardware, DFT would increase power consumption and computational complexity. D. S. Zrnic [13] illustrated that, when the power spectrum of the signal is symmetrical, the pulse-pair method is unbiased for Doppler shift estimation. Key [14] proposed a linear phase estimator. Based on this, Fitz [15] developed a frequency estimator, which is applicable to problems in communications requiring high speed, recursive frequency estimation. Nevertheless, the methods presented above [13-15] except the pulse-pair method are rarely used in BBADCP due to their flaws of estimation accuracy or computational complexity. This paper employs the pulse-pair method to estimate Doppler shift.

Although the pulse-pair method can obtain a higher precision Doppler shift quickly by extracting the phase information, the extracted phase can only fall within the range of $-\pi$ to $\pi$, as phase unwrapping occurs when the phase exceeds the range. By a controlled introduction of potential ambiguities, D. W. Tufts [16] provided a tabulation approach to solve phase ambiguity on the premise of phase unwrapping. Chen Jian [17] transmitted different frequencies signals to obtain different ambiguous frequencies, and then used a rectifying algorithm to correct the frequency shift of the two signals. When the frequencies of different echoes are corrected, the same frequency shift is the Doppler shift [18]. In recent years, N. Han [19] designed a combined signal for solving the ambiguity in current velocity measurement, discussed the design methods based on time delay and frequency, and improved the measurement accuracy. In addition, Chi Cheng $[8,20]$ proposed an orthogonal coprime signal, which is composed of two sub-signals with different frequencies and the signal length is prime to each other. Then, the robust Chinese remainder theorem (CRT) was used to relax the limit of the ambiguity velocity of conventional BBADCPs, while the complexity also increased. In this paper, we propose an orthogonal combined signal, and the corresponding processing method of echoes is designed, which can not only promote the velocity measurement accuracy but also decrease the computational complexity effectively.

The rest of this paper is organized as follows. Section 2 introduces the principle of Doppler velocity measurement, including the theory of pulse-pair method and the principle of phase wrapping. The design method of the proposed orthogonal combined signal and the corresponding echo processing method are described in Section 3. To further illustrate how to design the orthogonal combined signal, a design example is given in Section 4, and a conventional signal is also given for comparison. Section 5 presents the simulations of evaluating the performances of the proposed and the conventional methods. Finally, Section 6 concludes this paper.

\section{Theory}

The principle of Doppler current velocity measurement and the theory of the pulse-pair method is introduced in this section. The transmitted signal conventionally employed in BBADCPs and the corresponding phase wrapping are analyzed.

\subsection{Pulse-Pair Method}

Pulse-pair frequency estimation, which is measuring the phase of the received pulse for two consecutive pulses, is a widely adopted algorithm for its lower computation. Assuming that the complex of echo signal after adding white noise is

$$
y(t)=x(t)+n(t) \quad 0<t<T
$$

where $x(t)$ is echo signal with Doppler information and $n(t)$ is additive white Gaussian noise (AWGN) with a mean value of 0 , the autocorrelation of the echo signal can be expressed as: 


$$
R_{y}(\tau)=E\left[(x(t)+n(t)) \cdot(x(t+\tau)+n(t+\tau))^{*}\right]=R_{x}(\tau)+R_{n}(\tau)+R_{x n}(\tau)+R_{n x}(\tau)
$$

where $R_{x}(\tau)$ is the autocorrelation function of $x(t)$ and $R_{n}(\tau)$ is the autocorrelation function of noise $n(t) . x(t)$ is uncorrelated to $n(t)$, that is, $R_{x n}(\tau)=R_{n x}(\tau)=0, \quad R_{n}(\tau)=0(\tau \neq 0), R_{y}(\tau)$ is the autocorrelation function of $y(t)$, which is the sum of two part autocorrelation function and can be expressed as:

$$
R_{y}(\tau)= \begin{cases}R_{x}(\tau)+R_{n}(\tau), & \tau=0 \\ R_{x}(\tau), & \tau \neq 0\end{cases}
$$

Thus, when $\tau \neq 0$, the autocorrelation function of the transmitted signal $R_{x}(\tau)$ can be estimated accurately and unbiased by the autocorrelation function of echo signal $R_{y}(\tau)$. According to Wiener-Khintchine theorem, the autocorrelation function and power spectrum function of a signal are a pair of Fourier transforms, that is:

$$
R_{x}(\tau)=A_{x}(\tau) \exp \left[j \phi_{x}(\tau)\right]=\int_{-\infty}^{+\infty} S_{x}(f) e^{j 2 \pi f_{c} \tau} d f
$$

where $A_{x}(t)$ is amplitude of autocorrelation function of transmitted signal $x(t)$, which is an even function of $\tau . \phi_{x}(\tau)$ is the phase information of the autocorrelation function of $x(t)$, which is an odd function of $\tau$. $S_{x}(f)$ is the power spectral density of $x(t)$. The first-order derivative of Equation (4) can be obtained as:

$$
R_{x}^{\prime}(\tau)=j 2 \pi \int_{-\infty}^{+\infty} f \cdot S_{x}(f) e^{j 2 \pi f_{c} \tau} d f
$$

Since the first moment of Gaussian white noise is constant, letting $\tau=0$, we have:

$$
R_{x}^{\prime}(0)=j 2 \pi \int_{-\infty}^{+\infty} f \cdot S_{x}(f) d f
$$

The average frequency of the signal, that is, the first moment estimation of the power spectrum, is expressed as:

$$
f_{x}=\frac{\int_{-\infty}^{+\infty} f \cdot S_{x}(f) d f}{\int_{-\infty}^{+\infty} S_{x}(f) d f}=\frac{R_{x}^{\prime}(0)}{j 2 \pi R_{x}(0)}=\frac{\phi^{\prime}(0)}{2 \pi}
$$

The above equation shows that the estimated frequency can be obtained by the derivative of the phase at $\tau=0$, which is extracted from the autocorrelation function of the complex signal. $\phi_{x}^{\prime}(0)$ is expressed as:

$$
\phi_{x}^{\prime}(0)=\lim _{x \rightarrow 0} \frac{\phi_{x}(\tau)-\phi_{x}(0)}{\tau}=\frac{\phi_{x}(\tau)}{\tau}
$$

Substituting Equation (8) into Equation (7), when $\tau=0, R_{y}(\tau)=R_{x}(\tau)$, the estimated average Doppler shift is [21]:

$$
\widehat{f}_{d}=f_{x}=\frac{\phi_{x}(\tau)}{2 \pi \tau}=\frac{1}{2 \pi \tau} \arctan \frac{\operatorname{Im}\left[R_{x}(\tau)\right]}{\operatorname{Re}\left[R_{x}(\tau)\right]},(\tau \rightarrow 0, \tau \neq 0)
$$

where the range of $\phi_{x}(\tau)$ is $[-\pi / 2, \pi / 2]$. According to the positive and negative property of $\operatorname{Im}\left(R_{x}(\tau)\right)$ and $\operatorname{Re}\left(R_{x}(\tau)\right)$, the range of $\phi_{x}(\tau)$ can be extended to $[-\pi, \pi]$, and $\phi_{x}(\tau)$ is rewritten as:

$$
\phi_{x}(\tau)=\left\{\begin{array}{cc}
\arctan \frac{I m\left[R_{x}(\tau)\right]}{R\left[R_{x}(\tau)\right]} & \operatorname{Re}\left[R_{x}(\tau)\right]>0 \\
\arctan \frac{\operatorname{Im}\left[R_{x}(\tau)\right]}{\operatorname{Re}\left(R_{x}(\tau)\right]}-\pi & \operatorname{Re}\left[R_{x}(\tau)\right]<0, \operatorname{Im}\left[R_{x}(\tau)\right]<0 \\
\arctan \frac{I m\left[R_{x}(\tau)\right]}{\operatorname{Re}\left[R_{x}(\tau)\right]}+\pi & \operatorname{Re}\left[R_{x}(\tau)\right]<0, \operatorname{Im}\left[R_{x}(\tau)\right]>0
\end{array}\right.
$$




\subsection{Phase Wrapping}

The relationship between current velocity and Doppler shift $\widehat{f}_{d}$ is as follows:

$$
\hat{v}=\frac{c}{2 f_{c}} \widehat{f}_{d}
$$

where $c$ is underwater sound velocity and $f_{c}$ is carrier frequency. Equation (11) can be rewritten by using Equation (9) as:

$$
\hat{v}=\frac{c}{4 \pi f_{c} \tau} \phi_{x}(\tau)
$$

According to Equation (9), the frequency estimation is directly related to the phase difference of autocorrelation function, and the phase difference is calculated by arctangent operation, thus this is the root of the phase wrapping. The value of arctangent function is in $[-\pi, \pi]$; from Equation (10), if the real phase difference exceeds this range, phase winding will occur, and then the estimated Doppler shift (current velocity) is incorrect. In this case, the real phase can be expressed as:

$$
\phi_{A}=\phi_{x}+2 n \pi
$$

where $\mathrm{n}$ is an integer. The real current velocity $v_{R}$ is the sum of estimated current velocity $\hat{v}$ and the ambiguity velocity of $V_{a m}$, from Equations (12) and (13), can be expressed as:

$$
v_{\mathrm{am}}=\frac{n c}{2 f_{c} \tau}
$$

To avoid the velocity ambiguity, conventional BBADCPs require the maximum measurable velocity should be less than or equal to the ambiguity velocity $\mathcal{V}_{a m}$ in advance of system deployment [7].

\section{Proposed Method}

This paper proposes an orthogonal combined signal for improving the estimated accuracy of current velocity in BBADCPs. The phase wrapping on each sub-signal can be eliminated or weakened by the transmitted signal design based on delay difference; the local coded signal is multiplied by the received echo signal so that the influence of autocorrelation sidelobe on current velocity measurement accuracy can be reduced. The details of our proposed method are given in this section.

\subsection{Orthogonal Combined Signal}

Phase wrapping will lead to velocity ambiguity, that is, the estimated current velocity is not accurate. From Equation (14), we can know that, if the integer $n$ can be estimated accurately, the problem of velocity ambiguity can be solved effectively. The common method is analyzing the echo signal or designing the transmitted signal to obtain the estimated value of integer $n$, such that the influence of ambiguity velocity can be eliminated. An orthogonal coprime signal is designed in [8]; the integer $n$ can be obtained by robust CRT. C. Chi [20] proposed an unwrapping combined signal that contains three pulses $y_{0}, y_{1}$, and $y_{2}$, where the time delay between pulses $y_{0}$ and $y_{1}$ is $\tau_{1}$, and the one between pulses $y_{1}$ and $y_{2}$ is $\tau_{2}$. Phase wrapping can be eliminated or weakened when $\tau_{1}, \tau_{2}$ satisfies Equation (15).

$$
\left\{\begin{array}{c}
\tau_{2}>\tau_{1} \\
\left(\tau_{2}-\tau_{1}\right)<2 \tau_{1} \\
\left(\tau_{2}-\tau_{1}\right)<2 \tau_{2}
\end{array}\right.
$$

According to Equation (15), we design an orthogonal combined signal and its corresponding echo signal processing method. The accuracy of velocity measurement is improved on the premise of, as far as possible, minimizing the amount of calculation. The structure of the proposed signal is shown in Figure 1. 


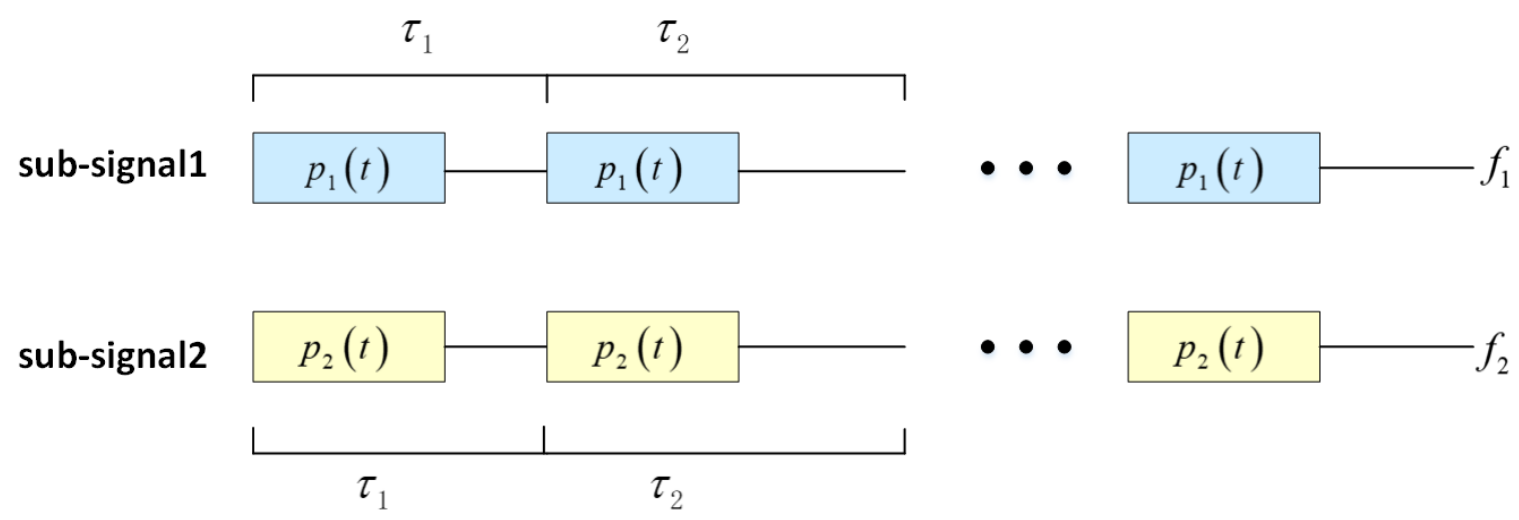

Figure 1. Structure of orthogonal combined signal.

The signal consists of two sub signals, Sub-Signals 1 and 2, with the same structure and equal length, and the spectrum is symmetric, to realize the orthogonality of the Sub-Signals 1 and 2. The carrier frequencies are $f_{1}$ and $f_{2}$, respectively. Both Sub-Signals 1 and 2 are composed of several combined coding signals of length $\tau_{1}+\tau_{2}$, The combined code signal of Sub-Signal 1 consists of code modulation signal $p_{1}(t)$ and a certain length blank time. Sub-Signal 2 is the same except the code modulation signal is $p_{2}(t)$. It should be noted that the lengths of $p_{1}(t)$ and $p_{2}(t)$ are equal. When the relationship between time delay $\tau_{1}$ and $\tau_{2}$ satisfies Equation (19), the problem of phase wrapping can be eliminated or weakened.

We denote the expressions of the transmitted pulses $p_{1}(t)$ and $p_{2}(t)$ of Sub-Signals 1 and 2 as follows:

$$
\begin{aligned}
& p_{1}(t)=a_{1}(t) \sin \left(2 \pi f_{1} t+\theta_{1}\right) \\
& p_{2}(t)=a_{2}(t) \sin \left(2 \pi f_{2} t+\theta_{2}\right)
\end{aligned}
$$

where $a_{1}(t)$ and $a_{2}(t)$ are two baseband coded pulses, which are uncorrelated and their lengths are equal. In BBADCPs, phase coding (such as Barker code, m sequence) and pulse frequency modulation are usually considered. We use phase coding in this paper; thus, $a_{1}(t)$ and $a_{2}(t)$ can be expressed as [8]:

$$
\begin{aligned}
& a_{1}(t)=\sum_{n_{1}=0}^{P_{1}-1} \sum_{h_{1}=1}^{L_{1}} \operatorname{rect}\left(\frac{\left(t-n_{1} T_{1}\right)-l_{1} \tau_{10}}{\tau_{10}}\right) e^{j \varphi_{11} h} \\
& a_{2}(t)=\sum_{n=0}^{P_{2}-1} \sum_{l_{2}=1}^{L_{2}} \operatorname{rect}\left(\frac{\left(t-n_{2} T_{2}\right)-l_{2} \tau_{20}}{\tau_{20}}\right) e^{j \theta_{2} L_{2}}
\end{aligned}
$$

where $\operatorname{rect}(x)$ represents a rectangular window function, $L_{i}(i=1,2)$ is the number of symbols, $\tau_{i 0}(i=1,2)$ is the duration of each symbol, $\varphi_{l i}(i=1,2)$ is phase of the $l$ th code, $P_{i}(i=1,2)$ is the number of pulses used in $a(t), T_{i}(i=1,2)$ is the duration of one pulse, thus the duration of the transmitted signal is $T_{x}=P_{1} T_{1}=P_{2} T_{2}$.

\subsection{Echo Signal Processing}

Echo signals are real signals which can be expressed as:

$$
Y(t)=a_{1}(t) \sin \left(2 \pi\left(f_{1}+f_{d 1}\right) t+\theta_{1}\right)+a_{2}(t) \sin \left(2 \pi\left(f_{2}+f_{d 2}\right) t+\theta_{2}\right)+n(t)
$$

where $n(t)$ is white Gaussian noise and $f_{d 1}$ and $f_{d 2}$ are Doppler frequency shift measured by Sub-Signals 1 and 2, respectively. Using time window to segment the echo signal corresponding to the 
depth, the window length is $T_{w}$, which is usually equal to a depth cell, and satisfies $T_{w}=T_{i}(i=1,2)$. Thus, the echo signal of this depth is:

$$
y=\operatorname{rect}\left(\frac{t-t_{0}}{T_{x}}\right) Y(t)=W(t) Y(t)
$$

where $t_{0}$ is the start position of the time window. Since the pulse pair frequency estimation algorithm processes the complex signal, and the echo signal of ADCP is the real signal, it is necessary to complex the received signal through IQ modulation [22]. Two orthogonal signals multiply the received real signal $y(t)$, respectively, to obtain mixing signal $y_{1}(t)$ and $y_{2}(t)$, expressed as Equations (22) and (23). Mixing signal $y_{1}(t)$ is expressed as:

$$
\begin{aligned}
y_{1}(t) & =y(t) \times \sin \left(\omega_{c} t\right) \\
& =\frac{a_{1}(t) W(t)}{2}\left[\cos \left(\omega_{r 1} t-\omega_{c} t+\theta\right)-\cos \left(\omega_{r 1} t+\omega_{c} t+\theta\right)\right] \\
& +\frac{a_{2}(t) W(t)}{2}\left[\cos \left(\omega_{r 2} t-\omega_{c} t+\theta\right)-\cos \left(\omega_{r 2} t+\omega_{c} t+\theta\right)\right]+N_{s}(t)
\end{aligned}
$$

Mixing signal $y_{2}(t)$ is expressed as:

$$
\begin{aligned}
y_{2}(t) & =y(t) \times \cos \left(\omega_{c} t\right) \\
& =\frac{a_{1}(t) W(t)}{2}\left[\sin \left(\omega_{r 1} t+\omega_{c} t+\theta\right)-\sin \left(\omega_{r 1} t-\omega_{c} t+\theta\right)\right] \\
& +\frac{a_{2}(t)}{2}\left[\sin \left(\omega_{r 2} t+\omega_{c} t+\theta\right)-\sin \left(\omega_{r 2} t-\omega_{c} t+\theta\right)\right]+N_{c}(t)
\end{aligned}
$$

where $\omega_{r 1}=2 \pi\left(f_{1}+f_{d 1}\right) t=\left(\omega_{1}+\omega_{d 1}\right) t, \omega_{r 2}=2 \pi\left(f_{2}+f_{d 2}\right) t=\left(\omega_{2}+\omega_{d 2}\right) t, \omega_{c}=\pi\left(f_{1}+f_{2}\right)=$ $\left(\frac{\omega_{1}+\omega_{2}}{2}\right) t$ is the center frequency of signal, $N_{s}(t)=n(t) \times \sin \left(\omega_{c} t\right), N_{c}(t)=n(t) \times \cos \left(\omega_{c} t\right)$. Assuming that the low-pass filter is an ideal filter, the high-frequency part is filtered, and the low-frequency part $y_{1 p}(t), y_{2 p}(t)$ is obtained. The expression is as follows:

$$
\begin{aligned}
y_{1 p}(t) & =W(t)\left[\frac{a_{1}(t)}{2} \cdot \cos \left(\omega_{11} t-\omega_{c} t+\theta\right)+\frac{a_{2}(t)}{2} \cdot \cos \left(\omega_{r 2} t-\omega_{c} t+\theta\right)\right]+N_{s}(t) \\
& =W(t)\left\{\frac{a_{1}(t)}{2} \cdot \cos \left[\left(\omega+\omega_{d 1}\right) t+\theta\right]+\frac{a_{2}(t)}{2} \cdot \cos \left[\left(-\omega+\omega_{d 2}\right) t+\theta\right]\right\}+N_{s}(t) \\
l y_{2 p}(t) & =-W(t)\left[\frac{a_{1}(t)}{2} \cdot \sin \left(\omega_{r 1} t-\omega_{c} t+\theta\right)+\frac{a_{2}(t)}{2} \cdot \sin \left(\omega_{r 2} t-\omega_{c} t+\theta\right)\right]+N_{c}(t) \\
& =-W(t)\left\{\frac{a_{1}(t)}{2} \cdot \sin \left[\left(\omega+\omega_{d 1}\right) t+\theta\right]+\frac{a_{2}(t)}{2} \cdot \sin \left[\left(-\sigma+\omega_{d 2}\right) t+\theta\right]\right\}+N_{c}(t)
\end{aligned}
$$

where $\mathscr{\omega}=\left(\omega_{1}-\omega_{2}\right) / 2$. Let $\mu_{1}=\left(\mathscr{\omega}+\omega_{d 1}\right) t+\theta, \mu_{2}=\left(-\mathfrak{\omega}+\omega_{d 2}\right) t+\theta$, the complex signal is:

$$
s(t)=W(t) \cdot\left(\frac{a_{1}(t)}{2} \cdot e^{-j \mu_{1}}+\frac{a_{2}(t)}{2} \cdot e^{-j \mu_{2}}\right)+N(t)
$$

Different from the traditional receiver processing method, in this paper, the complex signal is multiplied by $a_{1}(t)$ and $a_{2}(t)$, respectively. We can obtain a new signal, and then use pulse-pair method to estimate the frequency of a new signal. The result of multiplication $s_{a 1}(t), s_{a 2}(t)$ is shown 
as follows:

$$
\begin{aligned}
s_{a 1}(t) & =W(t) \cdot\left(\frac{a_{1}(t)}{2} \cdot e^{-j \mu_{1}}+\frac{a_{2}(t)}{2} \cdot e^{-j \mu_{2}}+N(t)\right) \times a_{1}(t) \\
& =\frac{W(t)}{2} \cdot(\frac{e^{-j \mu_{1}}}{(i)}+\underbrace{a_{1}(t) \cdot a_{2}(t) \cdot e^{-j \mu_{2}}}_{(i i)}+N(t) \cdot a_{1}(t)) \\
s_{a 2}(t) & =W(t) \cdot\left(\frac{a_{1}(t)}{2} \cdot e^{-j \mu_{1}}+\frac{a_{2}(t)}{2} \cdot e^{-j \mu_{2}}+N(t)\right) \times a_{2}(t) \\
& =\frac{W(t)}{2} \cdot(\underbrace{a_{1}(t) \cdot a_{2}(t) \cdot e^{-j \mu_{4}}}_{(i i i)}+\underbrace{e^{-j \mu_{2}}}_{\left(n_{0}\right)}+N(t) \cdot a_{2}(t))
\end{aligned}
$$

According to Equations (27) and (28), after the processing, Parts (i) and (iv) are restored to a single frequency signal, and the sidelobe effect of the coded signal can be weakened after autocorrelation processing. Meanwhile, a new coded signal

$$
a_{1}(t) \cdot a_{2}(t)
$$

is generated. Let $a_{3}(t)=a_{1}(t) \cdot a_{2}(t)$, and the autocorrelation of $s_{a 1}(t)$ and $s_{a 2}(t)$ can be expressed as:

$$
\begin{aligned}
R_{1}(\tau) & =\int_{0}^{T_{1}} s_{a 1}(t) \cdot s_{a 1}^{*}(t+\tau) d t \\
& =R_{11}+R_{13}+R_{1 N}+R_{31}+R_{33}+R_{3 N} \\
R_{2}(\tau) & =\int_{0}^{T_{1}} s_{a 2}(t) \cdot s_{a 2}^{*}(t+\tau) d t \\
& =R_{22}+R_{24}+R_{2 N}+R_{42}+R_{44}+R_{4 N}
\end{aligned}
$$

where subscripts correspond to Items (i)-(iv) of Equations (27) and (28), respectively. $R_{11}$ denotes autocorrelation between (i) and (i), $R_{13}$ denotes autocorrelation between (i) and (iii), and so on. It is worth noting that the sum of the autocorrelation operation of (i) $R_{11}$ and (ii) $R_{22}$ is equivalent to the result of the autocorrelation of the complex received signal $s(t)$ directly in the conventional method, the results are affected by the sidelobe of the coded signal.

We only obtain two items similar to (ii)-(iii) correlation results in the conventional processing method. The purpose of introducing local signal $a_{1}(t), a_{2}(t)$ here is to eliminate the influence of sidelobe after autocorrelation of one coded signal.

Since $a_{1}(t)$ and $a_{2}(t)$ are uncorrelated, and Gaussian white noise and signal are orthogonal, Equations (29) and (30) can be simplified as:

$$
\begin{aligned}
& R_{1}(\tau)=R_{11}+R_{33} \\
& R_{2}(\tau)=R_{22}+R_{44}
\end{aligned}
$$

In particular, when $a_{1}(t)$ and $a_{2}(t)$ are the same code signal, Equation (28) can be omitted, and the result of autocorrelation is $R(\tau)=R_{11}+R_{22}$. When $a_{1}(t)$ and $a_{2}(t)$ are coded signals with equal number of symbols and an equal bandwidth, from Equations (31) and (32), we can see that two items $R_{22}$ and $R_{44}$ are generated, which is equivalent to two more measurements, thus improving the accuracy of the current measurement.

Comparing with the combined signal [19], the orthogonal combined signal has higher accuracy in current velocity measurement. Besides, comparing with orthogonal coprime signal $[8,20]$, orthogonal combined signal omits the steps of designing the coprime length of signal and resolving the velocity 
ambiguity, which can reduce the amount of computation. The proposed signal is easier to design than orthogonal coprime signals and theoretically has higher precision.

\section{Design Example of the Orthogonal Combined Signals}

In this section, we give the design examples of the proposed signal and the conventional signal. Considering the center frequency of proposed signal and conventional signal is $f_{0}=600 \mathrm{kHz}$, bandwidth is $B_{0}=100 \mathrm{kHz}$. When using the conventional signal, the length of a single coding pulse is $1 / 200,000=0.02 \mathrm{~ms}$. We use the coded signal modulated by fifth-order $\mathrm{m}$-sequence in this design. Besides, to simulate the underwater channel environment, zero-mean white Gaussian noise with variance $\sigma^{2}$ is added. The sound speed in water is set to $1500 \mathrm{~m} / \mathrm{s}$. The Doppler frequency shift is $2400 \mathrm{~Hz}$, and the real current velocity is $3 \mathrm{~m} / \mathrm{s}[8,19,20]$. The transducer is oriented vertically downward, thus the multipath problem can be ignored. Measurement depth is $0-20 \mathrm{~m}$ and the layer thickness is $0.75 \mathrm{~m}$.

We briefly run through the design considerations and selected parameters of signals used in simulation, including conventional signals and proposed signals. Firstly, we introduce the design method of the proposed signal. Two sub-signals $p_{1}(t)$ and $p_{2}(t)$ are required in our proposed orthogonal combined signal; we set the center frequencies of $p_{1}(t)$ and $p_{2}(t)$ as $f_{1}=575 \mathrm{kHz}$ and $f_{2}=625 \mathrm{kHz}$, respectively. The bandwidthd $B_{1}$ and $B_{2}$ of $a_{1}(t)$ and $a_{2}(t)$ used in $p_{1}(t)$ and $p_{2}(t)$ are both $50 \mathrm{kHz} . p_{1}(t)$ and $p_{2}(t)$ are in two separate subbands and hence they are orthogonal. It should be noted that $a_{1}(t)$ and $a_{2}(t)$ can be two identical PN codes or two different PN codes, but the number of symbols should be the same. In this design, two different types of PN codes with the same length are selected, m-sequence and gold-sequence. Both types of PN codes are widely used in communication field due to their sharp autocorrelation peaks.

In our design, $a_{1}(t)$ is a fifth-order m-sequence and $a_{2}(t)$ is a fifth-order gold-sequence. The number of symbols is $2^{5}-1=31$ for both, the length of a single code pulse is $1 / 100,000=0.04 \mathrm{~ms}$, and time duration of both coded signals is $31 \times 0.04=1.24 \mathrm{~ms}$, repeating one time. Based on time delay relation of phase unwrapping in Equation (15), we set $\tau_{1}=2.1 \mathrm{~ms}$ and $\tau_{2}=2.2 \mathrm{~ms}$ (that is, the blank time is during 0.86 and $0.96 \mathrm{~ms}$, respectively). The duration of the whole coded signal should be $2.1+2.2=4.3 \mathrm{~ms}$.

For comparison, fifth-order m-sequence is used as the coded signal $a_{0}(t)$, the symbols number of one m-sequence is $2^{5}-1=31$, the duration of a single-coded signal is $31 \times 0.02=0.62 \mathrm{~ms}$, it is repeated $4.30 / 0.62 \approx 7$ times, and the duration of whole coded signal is $0.62 \times 7=4.34 \mathrm{~ms}$. These setup parameters of the two sub-signals are chosen to guarantee that our proposed orthogonal combined signal has the same center frequency and bandwidth as the conventional signal, and thus they are comparable.

The normalized autocorrelation function of the conventional signal and the proposed signal is shown in Figure 2, which also shows the time resolution of the two signals. In the design of the transmitted signal of ADCP, we hope that the autocorrelation function of the signal has a sharp correlation peak and a small sidelobe. In Figure 2a, the autocorrelation function of the conventional signal has many high energy side peaks, the envelope of main peak and side peak is a triangle, and its width is much longer than the proposed signal. The proposed signal has a sharp correlation peak similar to the conventional signal and the sidelobe is very small and uneven distribution, which is more conducive to obtain high ranging accuracy and range resolution. 


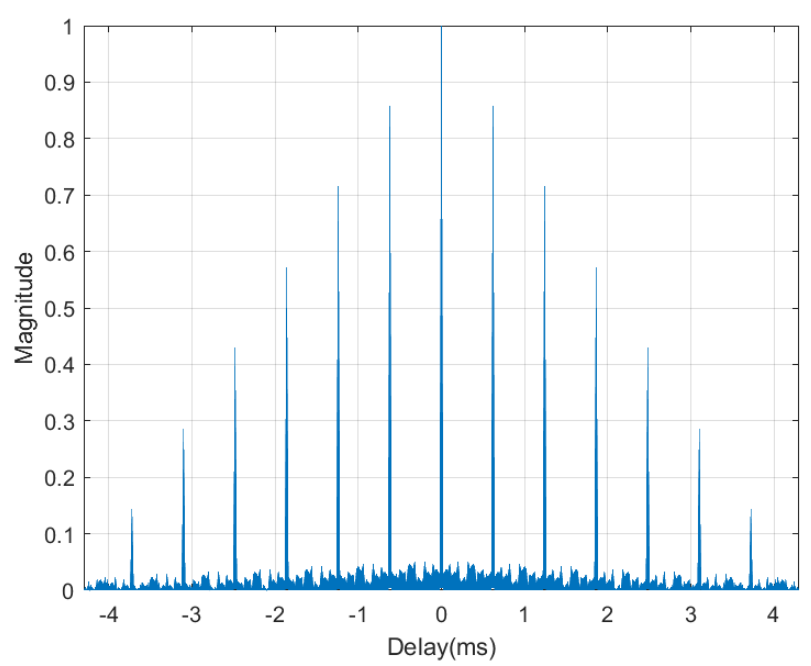

(a)

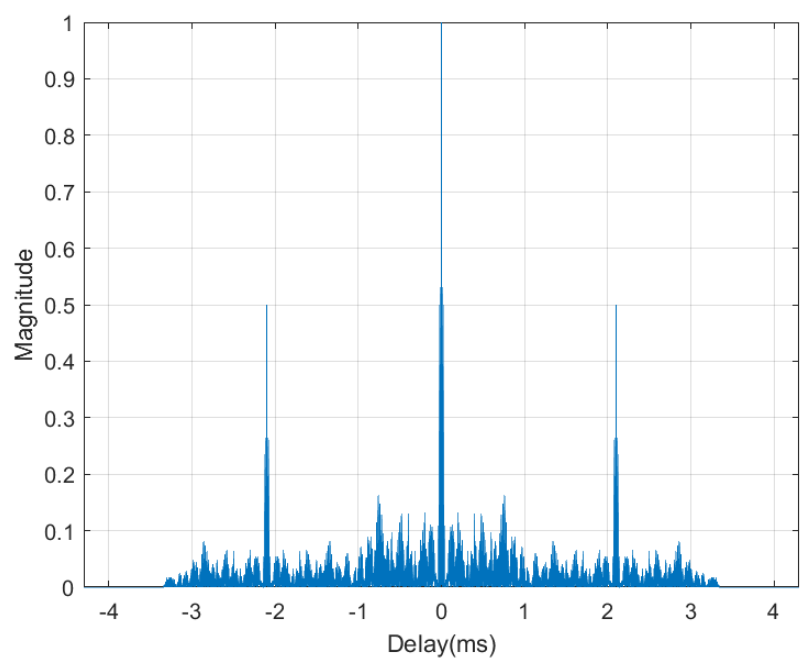

(b)

Figure 2. Autocorrelation functions of conventional signal and orthogonal combined signal: (a) autocorrelation function of conventional signal; and (b) autocorrelation function of proposed signal.

\section{Simulation}

The performance of the proposed method and the conventional signal are evaluated in this section. The current velocity estimated accuracy is compared under different SNRs and current velocities. The conventional signal is a sine wave modulated by m-sequence as shown in Section 4, which is obtained by Equation (16).

Standard deviation can reflect the dispersion of a set of data objectively and accurately. Suppose the estimated velocity of $i$ th measurement is $\widehat{v}_{i}$; then, the standard deviation an of $N$ times measurement results can be expressed as:

$$
S T D_{v}=\sqrt{\frac{1}{N-1} \sum_{i=1}^{N}\left(\hat{v}_{i}-\bar{v}_{e s t}\right)^{2}}
$$

where $\bar{v}_{\text {est }}$ is the average current velocity of $\mathrm{N}$ times measurements; the smaller is the $S T D_{v}$, the more stable are the data. In this paper, each standard deviation shown in a figure is obtained by calculating the velocity estimates from 50 trials $(n=50)$ at the same depth. 
First, the echo signals in different SNR are shown in Figures 3 and 4. The SNR decreases from Figures $3 \mathrm{~b}-\mathrm{e}$ and $4 \mathrm{~b}-\mathrm{e}$ and signals are nearly submerged in noise at SNR of $0 \mathrm{~dB}$. The performance comparison of the proposed signal and the conventional signal under the in-band SNR from 0 to $30 \mathrm{~dB}$ is shown in Figure 5, and the results of the numerical simulation are taken by 10 tests. It shows that the average value of velocity estimated by 10 tests under different SNR is $3 \mathrm{~m} / \mathrm{s}$. It can be seen that the proposed signal can be accurately estimated above SNR of $10 \mathrm{~dB}$. We also observe that the estimation result of the proposed signal at $\mathrm{SNR}$ of $6 \mathrm{~dB}$ is almost equivalent to that of the conventional signal at SNR of $14 \mathrm{~dB}$. That is, compared with the conventional method, the SNR gain of $8 \mathrm{~dB}$ can be achieved by our method. However, when SNR is lower than $6 \mathrm{~dB}$, the error of the proposed method increases.

(a)

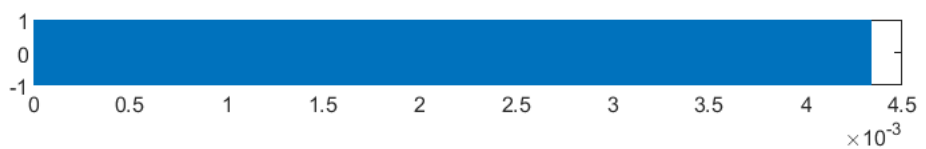

(b)

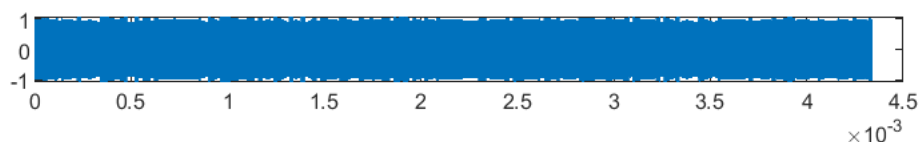

(c)

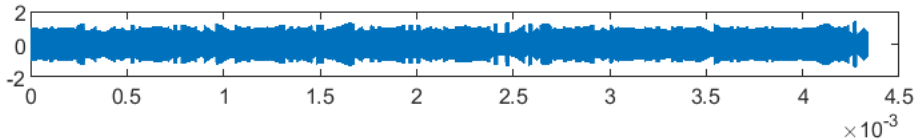

(d)

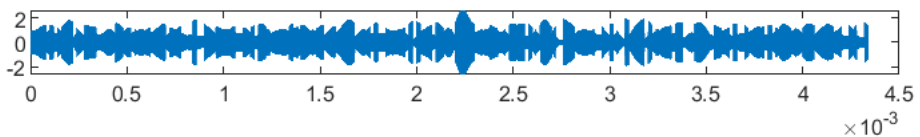

(e)

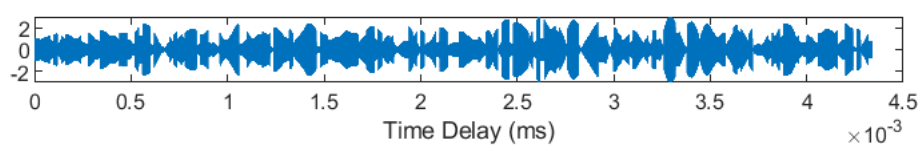

Figure 3. The echo signals of conventional method under different inband SNR: (a) Pure echo signal; (b) echo signal at $S N R=25 \mathrm{~dB}$; (c) echo signal at $S N R=15 \mathrm{~dB}$; (d) echo signal at $S N R=5 \mathrm{~dB}$; and (e) echo signal at $S N R=0 \mathrm{~dB}$.

(a)

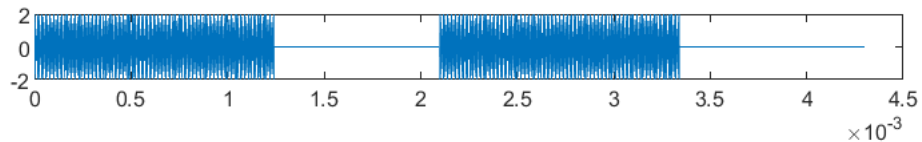

(b)

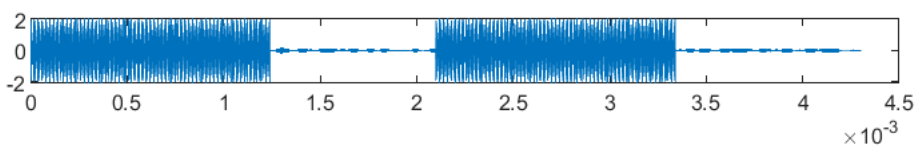

(c)

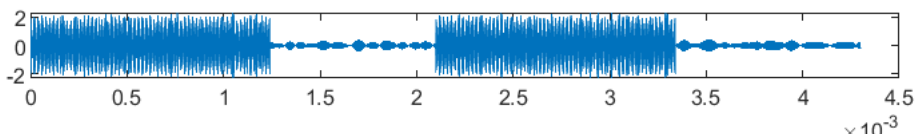

(d)

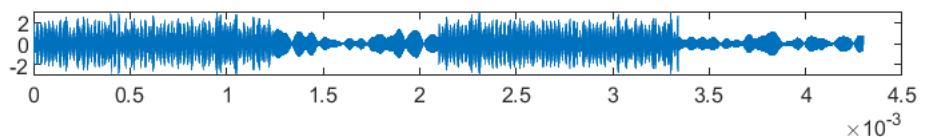

(e)

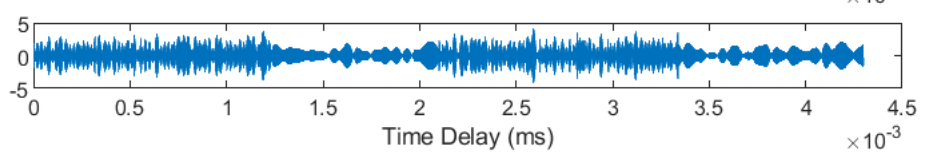

Figure 4. The echo signals of proposed method under different inband SNR: (a) Pure echo signal; (b) echo signal at $S N R=25 \mathrm{~dB}$; (c) echo signal at $S N R=15 \mathrm{~dB}$; (d) echo signal at $S N R=5 \mathrm{~dB}$; and (e) echo signal at $S N R=0 \mathrm{~dB}$. 
Figure 6 is a comparison of the current velocity estimation results of each measurement sample when the in-band SNR is $15 \mathrm{~dB}$; the numerical simulation concludes 50 tests. Setting real current velocity as $3 \mathrm{~m} / \mathrm{s}$, we can see that the value of current velocity measured by conventional signal fluctuates violently, and the average value of multiple measurements is about $3 \mathrm{~m} / \mathrm{s}$, but the measurement results are not stable. However, the value of current velocity measured by the proposed signal fluctuates in a small range around $3 \mathrm{~m} / \mathrm{s}$, which is more stable than the conventional signal.

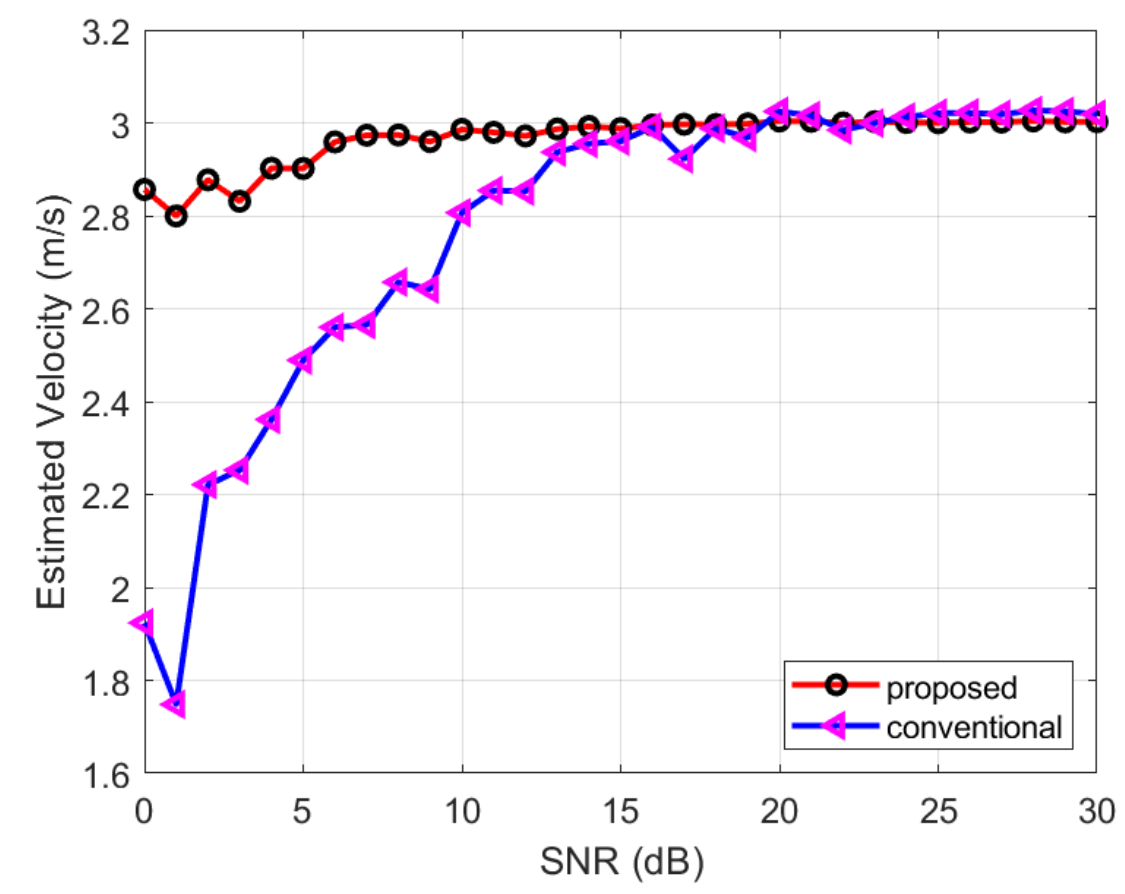

Figure 5. Performance comparison between the proposed signal and the convention signal under in-band SNR from 0 to $30 \mathrm{~dB}$.

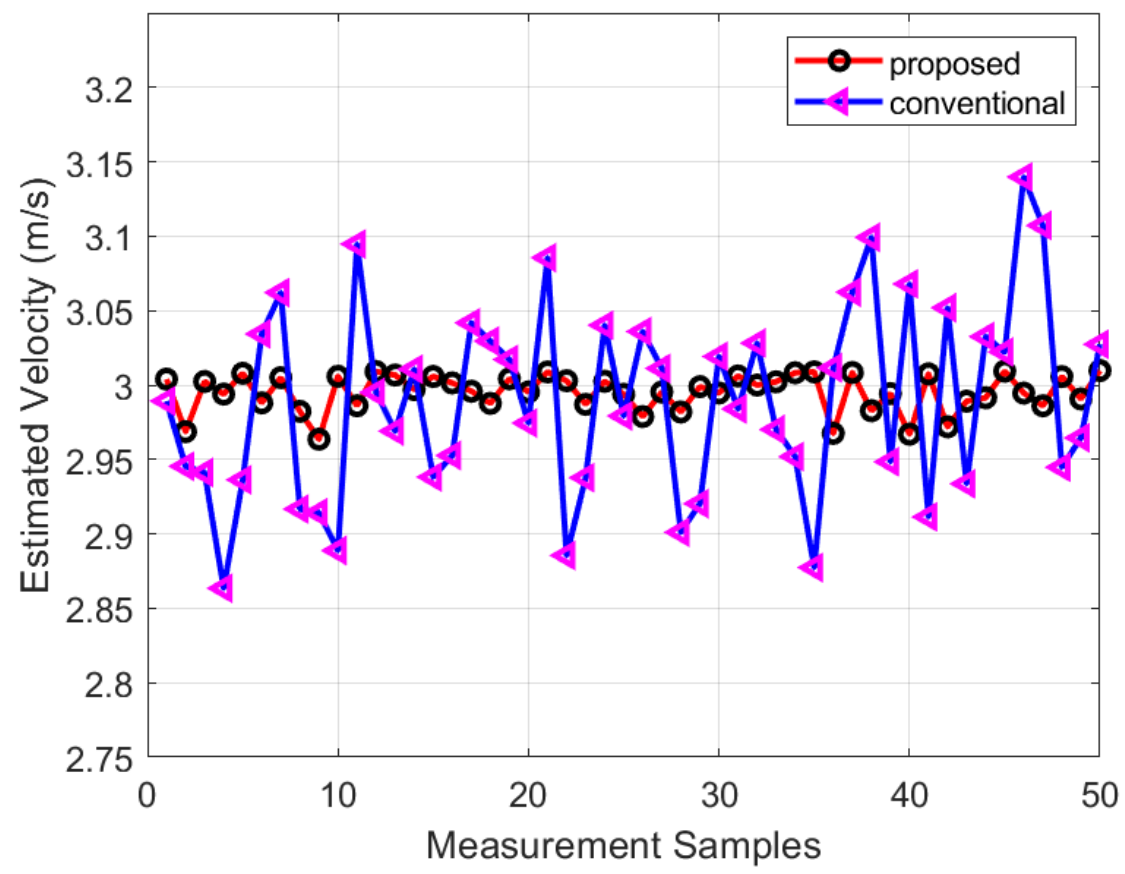

Figure 6. Comparison of current velocity estimation results of 50 measurement samples of two transmitted signals with SNR of $15 \mathrm{~dB}$. 
The comparison of the standard deviation of the estimated current velocity between the proposed signal and the conventional signal at the in-band SNR from 0 to $30 \mathrm{~dB}$ is shown in Figure 7. The standard deviation is from the result of 50 measurement samples. The standard deviation results show that the standard deviation of the proposed signal is less than the standard deviation of the conventional signal at the SNR from 0 to $30 \mathrm{~dB}$, and the maximum difference of standard deviation is about five times. Meanwhile, the value of the standard deviation decreases significantly with the increase of SNR. Compared with the conventional method, the proposed method is more stable and has better performance.

The standard deviation of current velocity estimation in different SNRs and current velocities $(0.5,3.5$, and $5 \mathrm{~m} / \mathrm{s})$ is shown in Figure 8. Figure $8 \mathrm{a}$ is the result of the conventional method and Figure $8 \mathrm{~b}$ is the result of the proposed method. It can be seen that, when using the proposed method, the lower is the real current velocity, the lower is the standard deviation of the estimated current velocity. The standard deviation of the estimated current velocity of the conventional method under the three kinds of current velocities are almost the same, which illustrates that the proposed signal has a better performance in the calmer flow environment.

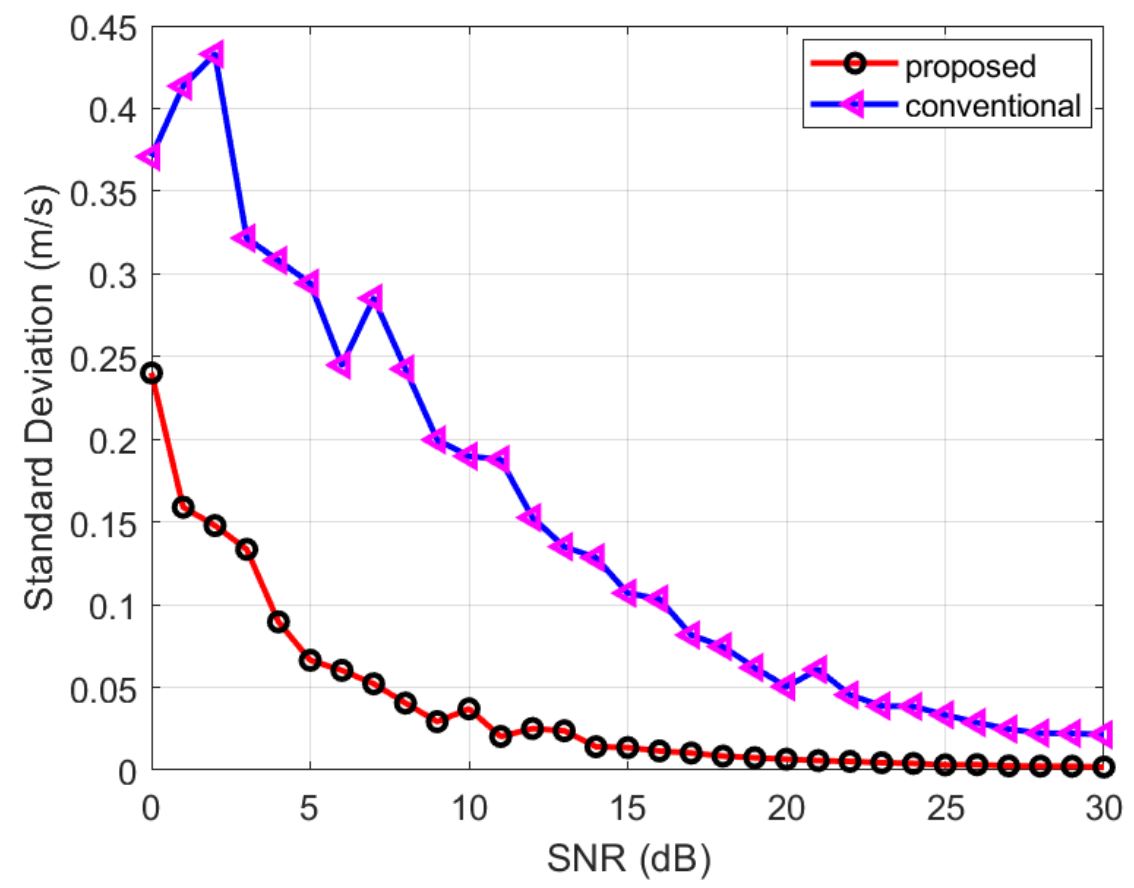

Figure 7. Comparison of standard deviation of 50 estimated current velocity with SNR of $15 \mathrm{~dB}$.

As shown in Figure 8a, when the SNR decreases, the estimation results of conventional methods become worse obviously. This is due to the sensitivity of the pulse-pair method, and the phase wrapping caused by noise disturbing the phase. Compared with Figure 8b, we can see that the proposed method obviously weakens the phase wrapping problem.

To guarantee the transmitted signal length is the same, the repeat times (RT) of a single coded signal is different between the conventional method and our method; the former is much greater than the latter. Figure 9 shows the effect of coding repetition; when the RT of a single coded signal increases, the measurement result becomes more stable. That is the measurement performance can be also improved by increasing the repeat times in our method.

It should be noted that, in the proposed method, the current velocity estimation is based on two sub-signals rather than a single signal as used in the convention method. To improve the accuracy of the proposed method, we need to perform two additional multiplication operations, and then use correlation functions and some simple operations to estimate the Doppler shift. As the echo signal processing method in Section 3.2. Therefore, our method is a little more complex than the conventional 
one. However, our method can save the steps of phase unwrapping to a certain extent. With the development of digital processor technology, the computational complexity caused by our method is easy to deal with. In addition, our method has more signal combination methods than the conventional method, which can generate more signal samples suitable for the actual situation and improve the accuracy of estimated current velocity.

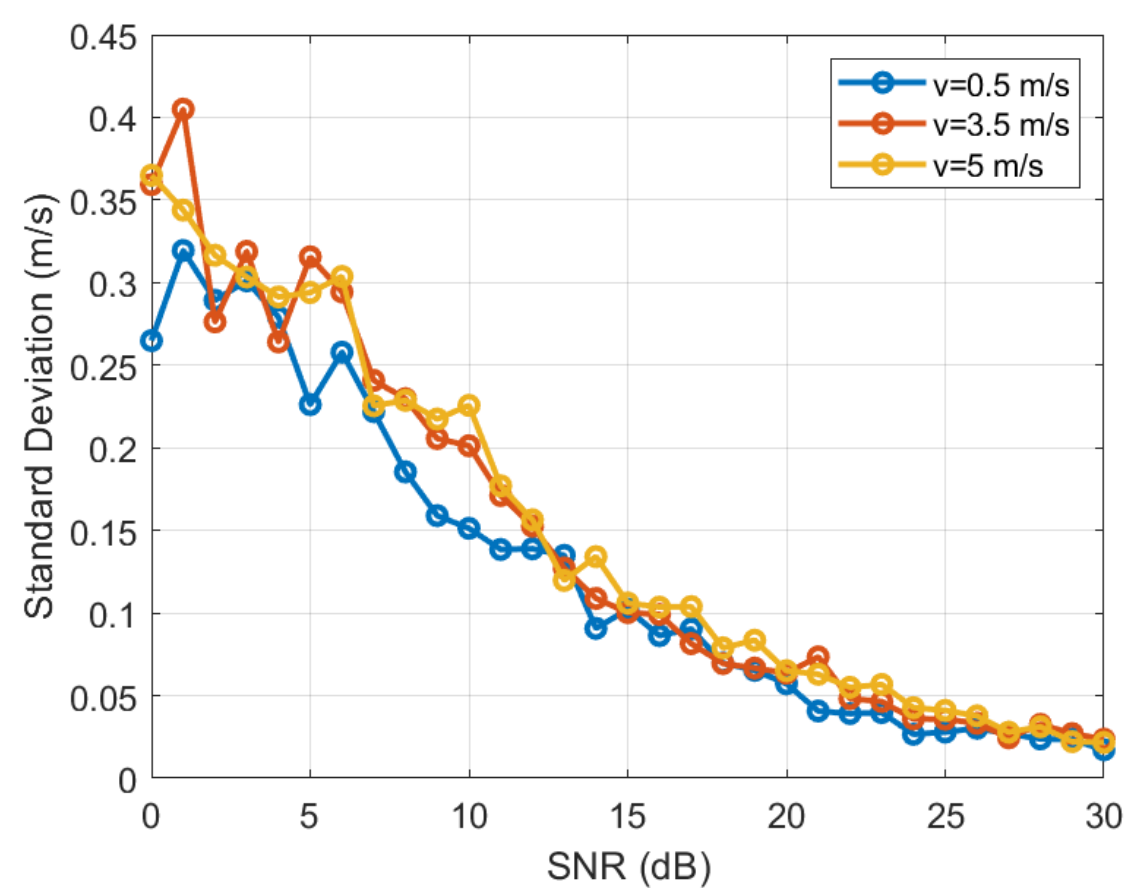

(a)

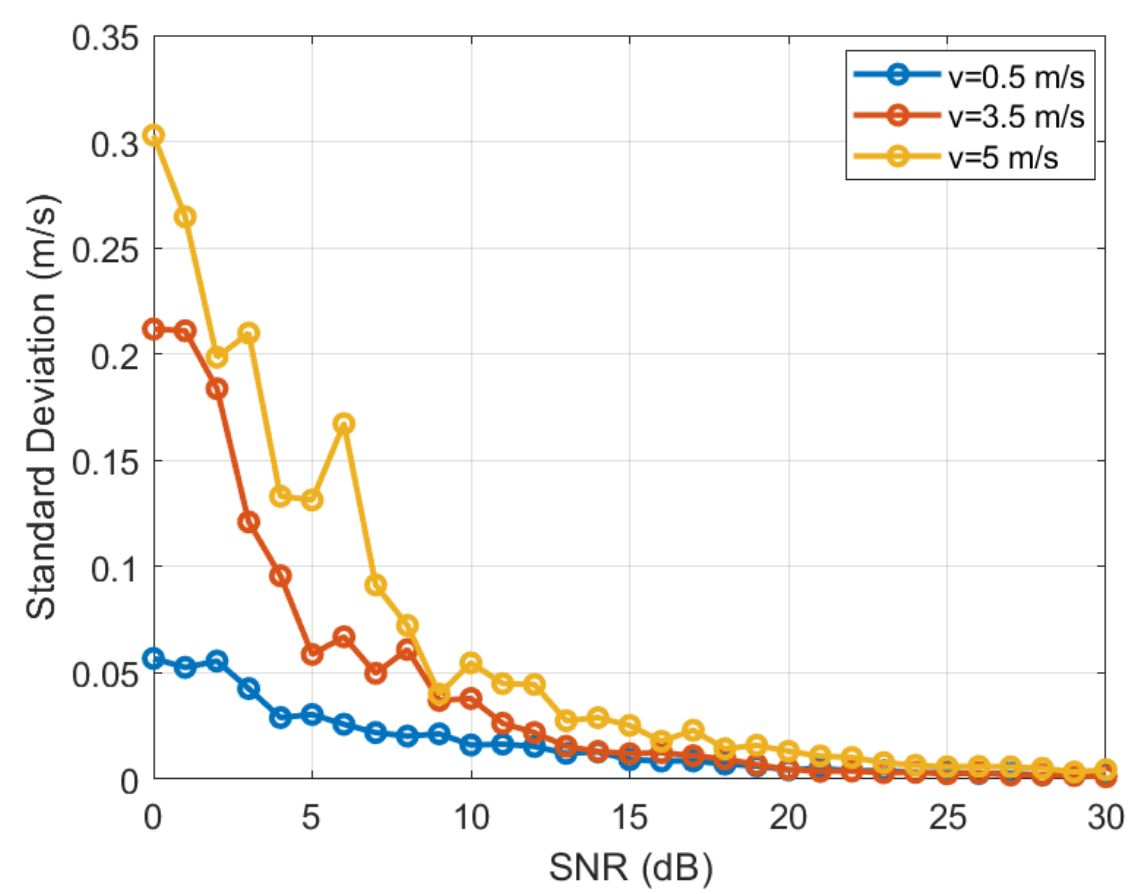

(b)

Figure 8. The standard deviation of estimated velocity under different SNR and different velocity values $(0.5 \mathrm{~m} / \mathrm{s}, 3.5 \mathrm{~m} / \mathrm{s}$ and $5 \mathrm{~m} / \mathrm{s})$ : (a) conventional signal; and (b) orthogonal combined signal. 


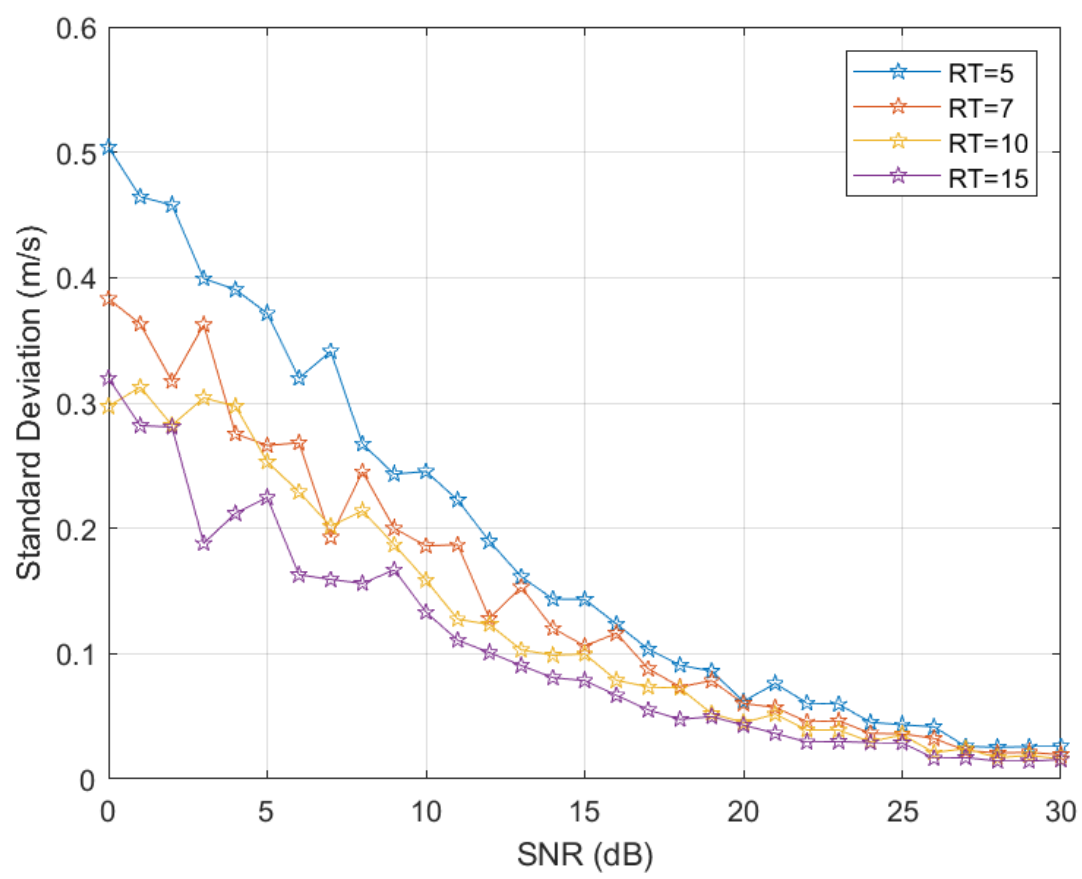

Figure 9. The effect of coding repetition on the performance of current velocity estimation.

\section{Conclusions}

In this paper, an orthogonal combined signal is proposed for BBADCPs, which breaks through the samples number limitation of conventional coded modulation signal and reduces the influence of phase wrapping by transmitted signal designed. Compared with the conventional method, this method can reduce the standard deviation to some extent, and generate up to $10 \mathrm{~dB}$ SNR gain. Besides, the method has the advantages of simple design and high measurement accuracy. The SNR gain and signal sample diversity obtained by our method can help to increase the configuration range of BBADCPs. In the future, the proposed method will be applied to the horizontal measurement, and the interference of the channel multipath effect will be considered. Besides, the sea experiment and the effect of the transducer angle are also the focus of our next work.

Author Contributions: Conceptualization, Y.L. and F.Y.; methodology, Y.L.; software, Y.L.; validation, Y.L. and F.Y.; formal analysis, Y.L. and F.Y; investigation, Y.L.; resources, E.C.; data curation, Y.L.; writing-original draft preparation, Y.L.; writing-review and editing, Y.L. and F.Y.; project administration, Y.F. and E.C.; funding acquisition, E.C. All authors have read and agreed to the published version of the manuscript.

Funding: This research was funded by National Natural Science Foundation of China (61771412 and 61871336) and the Fundamental Research Funds for the Central Universities (20720180068).

Conflicts of Interest: The authors declare no conflict of interest.

\section{References}

1. Prieur, F.; Hansen, R.E. Theoretical improvements when using the second harmonic signal in Acoustic Doppler Current Profilers. IEEE J. Ocean. Eng. 2013, 38, 275-284. [CrossRef]

2. Brumley, B.H.; Cabrera, R.G.; Deines, K.L.; Terray, E.A. Performance of a Broad-Band Acoustic Doppler Current Profiler. IEEE J. Ocean. Eng. 1991, 16, 402-407. [CrossRef]

3. Zedel, L.; Hay, A.E.; Cabrera, R.; Lohrmann, A. Performance of a single-beam pulse-to-pulse coherent Doppler profiler. IEEE J. Ocean. Eng. 1996, 21, 290-297. [CrossRef]

4. Appell, G.F.; Bass, P.D.; Metcalf, M.A. Acoustic Doppler Current Profiler performance in near surface and bottom boundaries. IEEE J. Ocean. Eng. 1991, 16, 390-396. [CrossRef]

5. Trevorrow, M.V.; Farmer, D.M. The use of Barker codes in Doppler sonar measurements. J. Atmos. Ocean. Technol. 1992, 9, 699-704. [CrossRef] 
6. Smith, J.A.; Pinkel, R. Improvement of Doppler estimation through repeat-sequence coding. In Proceedings of the OCEANS 91, USA/Ocean Technologies and Opportunities in the Pacific for the 90's, Honolulu, HI, USA, 1-3 October 1991; pp. 977-984.

7. Wanis, P.; Brumley, B.; Gast, J.; Symonds, D. Sources of measurement variance in broadband Acoustic Doppler Current Profilers. In Proceedings of the IEEE OCEANS 2010 MTS/IEEE SEATTLE, Seattle, WA, USA, 20-23 September 2010; pp. 1-5.

8. Chi, C.; Vishnu, H.; Beng, K.T.; Chitre, M. Utilizing orthogonal coprime signals for improving broadband Acoustic Doppler Current Profilers. IEEE J. Ocean. Eng. 2019, 99, 1-11. [CrossRef]

9. Dillon, J.; Zedel, L.; Hay, A.E.. On the Distribution of Velocity Measurements From Pulse-to-Pulse Coherent Doppler Sonar. IEEE J. Ocean. Eng. 2012, 37, 613-625. [CrossRef]

10. Miller, K.; Rochwarger, M. A covariance approach to spectral moment estimation. IEEE Trans. Inf. Theory 1972, 18, 588-596. [CrossRef]

11. Abeysekera, S. Performance of pulse-pair method of Doppler estimation. IEEE Trans. Aerosp. Electron. Syst. 1998, 34, 520-531. [CrossRef]

12. Ghogho, M.; Swami, A.; Durrani, T.S. Frequency estimation in the presence of Doppler spread: Performance analysis. IEEE Trans. Signal Process. 2001, 49, 777-789. [CrossRef]

13. Zrnic, D.S. Spectral Moment Estimates from Correlated Pulse Pairs. IEEE Trans. Aerosp. Electron. Syst. 1977, AES-13, 344-354. [CrossRef]

14. Xiao, Y.C.; Wei, P.; Xiao, X.C.; Tai, H.M. A fast and accurate single frequency estimator. Electron. Lett. 2004, 40, 910-911. [CrossRef]

15. Fitz, M.P. Further results in the fast estimation of a single frequency. IEEE Trans. Commun. 1994, 42, 862-864. [CrossRef]

16. Tufts, D.W.; Fiore, P.D. Simple, effective estimation of frequency based on Prony's method. In Proceedings of the 1996 IEEE International Conference on Acoustics, Speech, and Signal Processing, Atlanta, GA, USA, 9 May 1996; Volume 5, pp. 2801-2804.

17. Jian, C.; Shen, Z.; Zhigang, H.; Chunjie, Q. Acoustic velocity measurement in seawater based on phase difference of signal. In Proceedings of the IEEE 2011 10th International Conference on Electronic Measurement and Instruments, Chengdu, China, 16-19 August 2011; pp. 181-184.

18. Ellis, D.; Washburn, L.; Ohlmann, C.; Gotschalk, C. Improved methods to calculate depth-resolved velocities from glider-mounted ADCPs. In Proceedings of the 2015 IEEE/OES Eleveth Current, Waves and Turbulence Measurement (CWTM), St. Petersburg, FL, USA, 2-6 March 2015; pp. 1-10.

19. Han, N.; Fan, Z.; Fang, S. Phase unwrapping methods for solving the ambiguity in current velocity estimation based on combined signal design. Flow Meas. Instrum. 2018, 59, 126-134. [CrossRef]

20. Chi, C.; Vishnu, H.; Beng, K.T. Improving Broadband Acoustic Doppler Current Profiler with Orthogonal Coprime Pulse Pairs and Robust Chinese Remainder Theorem. In Proceedings of the 2018 OCEANS-MTS/IEEE Kobe Techno-Oceans (OTO), Kobe, Japan, 28-31 May 2018; pp. 1-5.

21. Trump, C.L.; Allan, N.; Marmorino, G.O. Side-looking ADCP and Doppler radar measurements across a coastal front. IEEE J. Ocean. Eng. 2000, 25, 423-429. [CrossRef]

22. Rao, Z.-Y.; Feng, C.-Y. Implementation of coded pulse complex correlation algorithm on broadband ADCP. In Proceedings of the 2011 International Conference on Consumer Electronics, Communications and Networks (CECNet), XianNing, China, 16-18 April 2011; pp. 410-413.

(C) 2020 by the authors. Licensee MDPI, Basel, Switzerland. This article is an open access article distributed under the terms and conditions of the Creative Commons Attribution (CC BY) license (http://creativecommons.org/licenses/by/4.0/). 